\title{
Budd-Chiari Like Syndrome Associated with a Primary Pulmonary Adenocarcinoma in a Dog
}

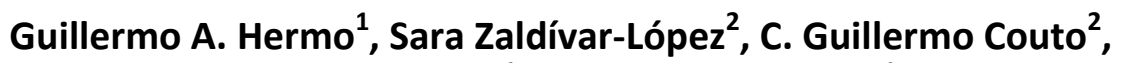 \\ Daniel F. Alonso ${ }^{1}$ and Daniel E. Gomez ${ }^{1}$ \\ ${ }^{1}$ Laboratory of Molecular Oncology, Department of Science and Technology, Quilmes National \\ University, Saenz Peña, Buenos Aires, Argentina \\ ${ }^{2}$ Oncology and Hematology Service, Department of Veterinary Clinical Sciences, College of \\ Veterinary Medicine, the Ohio State University, Columbus, $\mathrm{OH}$, United States
}

Correspondence should be addressed to: Guillermo A. Hermo; ghermo@unq.edu.ar

Received 28 May 2013; Accepted 24 June 2013; Published 24 October 2013

Academic Editor: Mehmet Haligür

Copyright @ 2013 Guillermo A. Hermo, Sara Zaldívar-López, C. Guillermo Couto, Daniel F. Alonso and Daniel E. Gomez. Distributed under Creative Commons CC-BY 3.0

\begin{abstract}
A 10-year-old female crossbred dog of $6 \mathrm{~kg}$ bodyweight was presented with signs of marked abdominal distention. The abdominal skin vasculature appeared prominent. After an exhaustive physical examination, laboratory analysis (table 1) and imaging studies, the case was diagnosticated as Budd-Chiari like syndrome due to lung adenocarcinoma. Abdominal ultrasonography revealed a neoplasia of $4 \mathrm{~cm}$ in diameter with irregular borders located in right accessory lung in contact with the caudal vena cava and surface thorax of the diaphragm. Immediately after sonographic diagnosis, the canine was admitted to intensive care and hemodynamically stabilized and submitted to surgery after $24 \mathrm{hrs}$. Then we made a exploratory thoracotomy with intention of achieve a lung lobectomy. After surgery chemotherapy with doxorrubicin $30 \mathrm{mg} / \mathrm{m} 2$ was administered. The objective of our study was to describe the image and pathological data findings in a dog with Budd-Chiari like syndrome; including sonographic findings, treatment and its response, in a syndrome that rarely affect small animals. Although no data are available on incidence of this disease in veterinary medicine, there is no doubt that this is a very serious illness despite its rare appearance. To our knowledge, this is one of the few cases where BCLS is reported associated to lung adenocarcinoma in veterinary medicine.
\end{abstract}

Keywords: Cancer, canine, tumor, compression.

\section{Introduction}

Budd-Chiari syndrome (BCS) is an uncommon disorder characterized by the thrombotic or non-thrombotic obstruction of hepatic venous outflow. ${ }^{1}$ This obstruction can be found anywhere along the hepatic venous system, from the hepatic veins to the caudal vena cava (CVC)-right atrial junction. Hepatic veno occlusive disease and cardiac disorders are excluded from this definition. BCS is a heterogeneous clinical condition: it can be either curable or potentially lethal. Patients with BCS have good prognosis with appropriate management, compared to

Cite this Article as: Guillermo A. Hermo, Sara Zaldívar-López, C. Guillermo Couto, Daniel F. Alonso and Daniel E. Gomez (2013), "Budd-Chiari Like Syndrome Associated with a Primary Pulmonary Adenocarcinoma in a Dog," International Journal of Veterinary Medicine: Research \& Reportss, Vol. 2013 (2013), Article ID 594516, DOI: 10.5171/2013.594516 
other chronic liver diseases. BCS is a rare but important syndrome, since many malignant disorders may result in BCS.

This syndrome is named based on the two individuals that first documented it: George Budd, a British internist, who described three cases of hepatic vein thrombosis due to abscess-induced phlebitis in 1845; and Hans Chiari, an Austrian pathologist, who first described the pathologic findings of BCS as "liver with obliterating endophlebitis of the hepatic veins" in 1899.

Currently there are no accurate data and consensus on prevalence/incidence in BCS in people ${ }^{1,2}$; although one study reports a prevalence of $1 / 100,000$ people worldwide. ${ }^{1}$ In veterinary medicine there is scarce information regarding prevalence of this disease. BCLS (like) was reported in 10 dogs with cor triatriatum dexter, ${ }^{3-6}$ and in dogs with intracardiac tumors.7, 8 One case report described CVC obstruction and resultant high protein ascites, due to an intraluminal leiomyosarcoma and another one an adrenal pheochromocytoma resulting in BCLS in dogs. ${ }^{9,} 10$

\section{Case Report}

A 10-year-old female mixed-breed female dog was presented to Clinica Veterinaria del Sur (Buenos Aires, Argentina) for evaluation of polydipsia and sudden-onset abdominal distention over the previous 7 days.

On physical examination, the dog weighed $6 \mathrm{~kg}$; she had mild dyspnea and marked abdominal distention associated with peritoneal effusion. The cutaneous abdominal vasculature was prominent. The dog also showed anorexia, lethargy, lumbar pain and edema in hindlimbs. Cardiac auscultation and ECG was normal, and there was no jugular vein distention. Further evaluation included a complete blood count, serum biochemistry panel, abdominocentesis (with fluid analysis) (table 1), electrocardiogram, echocardiography, and abdominal and thoracic ultrasonography. Urinalysis revealed marked proteinuria on dipstick (Multistix 10 Sg Urine Test Strips, Siemens ${ }^{\circledR}$, Buenos Aires, Argentina).

Table 1. Laboratory Results

\begin{tabular}{|c|c|c|c|}
\hline Hematology & Units & $\%$ & Value \\
\hline Packed cell volume & $\%$ & & 30 \\
\hline Hemoglobin & $\mathrm{g} / \mathrm{dl}$ & & 7.5 \\
\hline Red blood cell (RBC) & value $\times 10^{12} / 1$ & & 3.3 \\
\hline White blood cell (WBC) & value $\times 10^{9} / 1$ & & 12.9 \\
\hline Band neutrophils & value $\times 10^{9} / 1$ & 4 & 0.516 \\
\hline Segmented neutrophils & value $\times 10^{9} / 1$ & 84 & 10.836 \\
\hline Eosinophils & value $\times 10^{9} / 1$ & 0 & 0 \\
\hline Basophils & value $\times 10^{9} / 1$ & 0 & 0 \\
\hline Lymphocytes & value $\times 10^{9} / \mathrm{l}$ & 9 & 1.161 \\
\hline Monocytes & value $\times 10^{9} / 1$ & 3 & 0.387 \\
\hline \multicolumn{2}{|l|}{ Chemistry profile } & Units & Value \\
\hline \multicolumn{2}{|l|}{ Urea } & $\mathrm{mg} / \mathrm{dl}$ & 55 \\
\hline \multicolumn{2}{|l|}{ Creatinine } & $\mathrm{mg} / \mathrm{dl}$ & 1.4 \\
\hline \multicolumn{2}{|l|}{ Glucose } & $\mathrm{mg} / \mathrm{dl}$ & 94 \\
\hline \multicolumn{2}{|l|}{ Serum total protein } & $\mathrm{g} / \mathrm{dl}$ & 5.5 \\
\hline \multicolumn{2}{|l|}{ Albumin } & $\mathrm{g} / \mathrm{dl}$ & 1,75 \\
\hline Total globulins & \multicolumn{2}{|c|}{$\mathrm{g} / \mathrm{dl}$} & 4.00 \\
\hline \multicolumn{2}{|c|}{ Albumin/Globulin ratio } & & 0.44 \\
\hline Alkaline phosphatase (ALP) & \multicolumn{2}{|c|}{$\mathrm{U} / \mathrm{l}$} & 815 \\
\hline
\end{tabular}




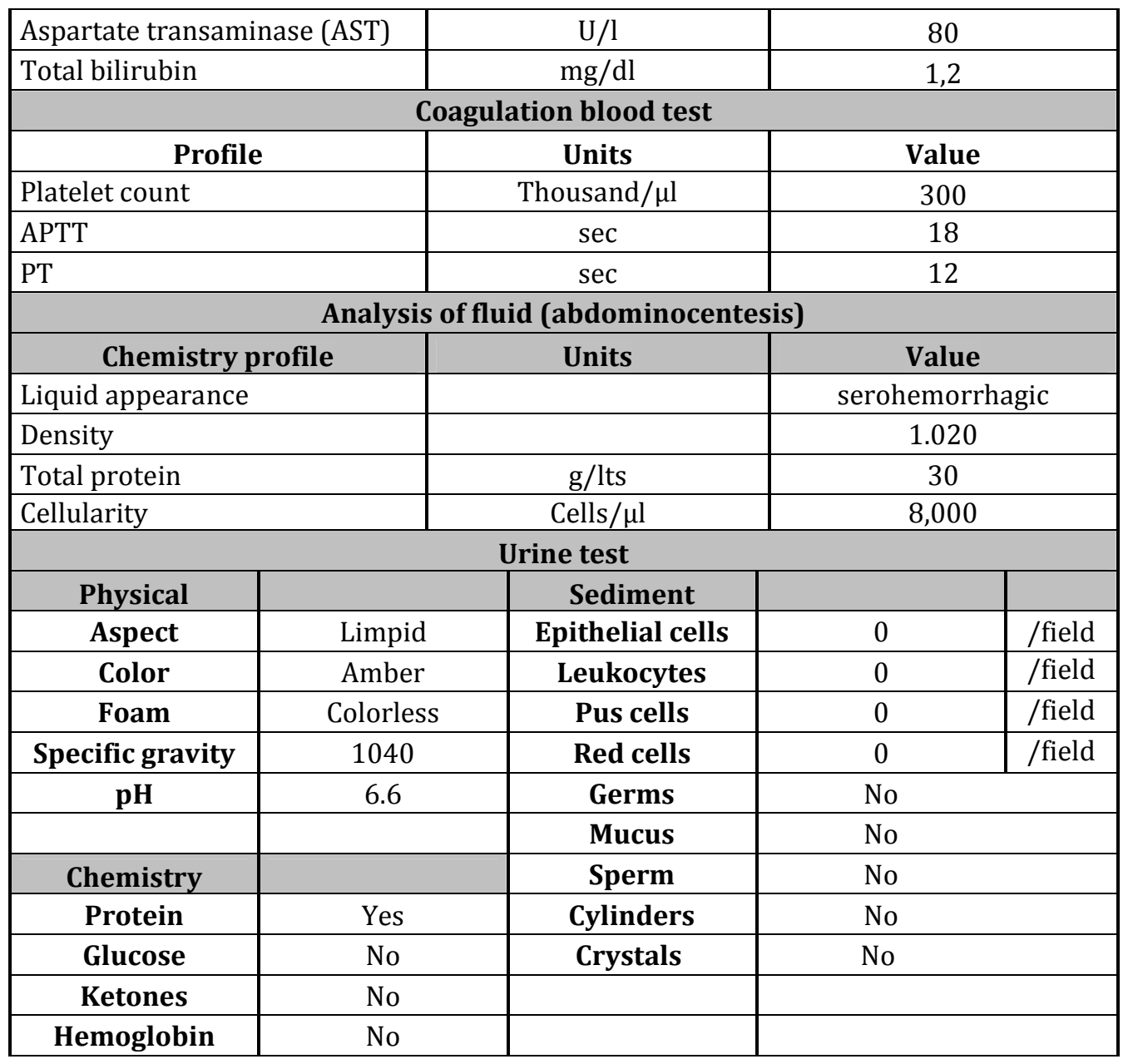

Conventional abdominal gray-scale ultrasonography revealed a 4-cm tumor with irregular borders, in direct contact with the CVC and thoracic surface of the diaphragm, what was later demonstrated to be large lung malignant tumor (Fig. 1 A, B). Dilation of the abdominal portion of the CVC, hepatomegaly, splenomegaly, abdominal effusion, and dilatation of the hepatic veins were also present. Color flow doppler studies showed absent, flat or hepatofugal flow in different parts of the hepatic veins, CVC, and intrahepatic collateral pathways, and slow portal vein flow. The distention of the CVC (in absence of heart failure) on gray-scale sonography and the previously mentioned clinical findings strongly suggested a diagnosis of BCLS. An exploratory thoracotomy was recommended. 


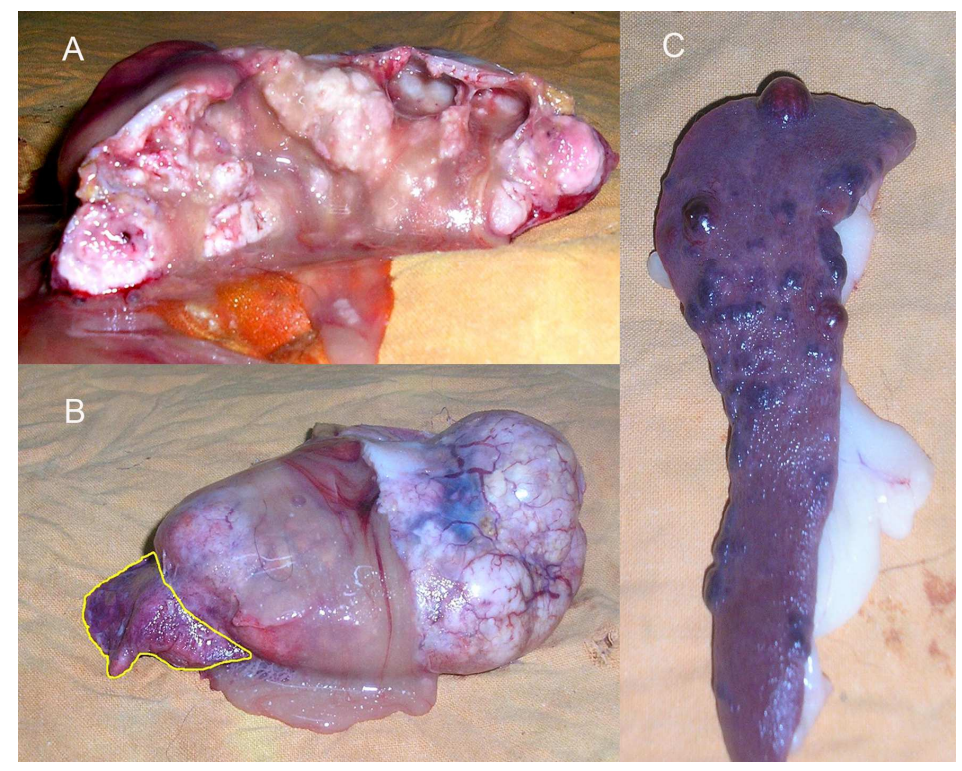

Fig. 1:- Macroscopic Images:

\section{A) The Samples were Obtained in the Necropsy. Lung Adenocarcinoma with Abundant Purulent Exudate.}

\section{B) The Samples were Obtained in the Necropsy. Left Lung Yellow Line Shows Normal Lung, the Rest is All Tumoral Tissue.}

\section{C) The Samples were Obtained in the Necropsy. Tumors in Spleen.}

The dog underwent surgery 24 hours later. After premedication with atropine sulphate (John Martin, Buenos Aires, Argentina), $0.04 \mathrm{mg} / \mathrm{kg}, \mathrm{SC}$, acepromazine maleate (Acedan, Holliday, Buenos Aires, Argentina) $0.03 \mathrm{mg} / \mathrm{kg} \mathrm{SC}$, and butorphanol (Torbutrol Plus, Fort Dodge, Buenos Aires, Argentina), $0.2 \mathrm{mg} / \mathrm{kg}$, IM, anesthesia was induced with sodium thiopental (Pentovet TM, Richmond, Buenos Aires, Argentina), 8 $\mathrm{mg} / \mathrm{kg}$, IV and maintained with isoflurane (IsoThesia; Butler Animal Health Supply, Dublin, OH, USA) and oxygen (IsoThesia; Butler Animal Health Supply, Dublin, OH, USA), delivered in a closed system.
An exploratory laparatomy and thoracotomy were done. During the thoracotomy, due to the large size of the tumor (11 x $6 \times 4 \mathrm{~cm})$, it was deemed unresectable, and an incisional biopsy was obtained. The abdominal fluid was drained, and biopsies of the spleen and liver were obtained for histopathological evaluation. Cytology of a fine needle aspirate of the lung tumor was compatible with a pulmonary carcinoma (Fig. 2 A). A histopathologic diagnosis of papillary pulmonary adenocarcinoma was made (Fig. 2 B). 


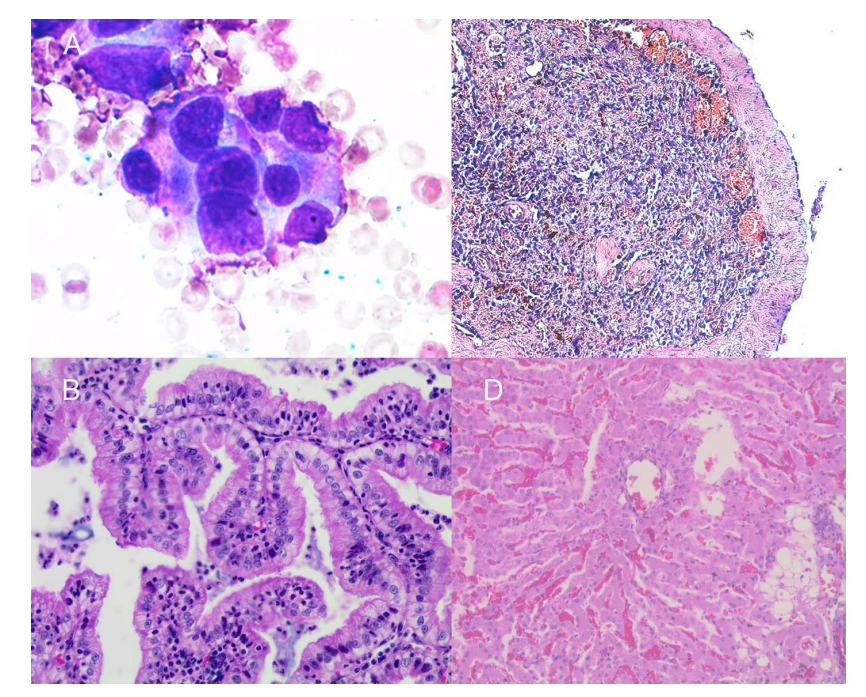

Fig. 2: - Microscopic Images:

A) Cytologic Sample Obtained by Fine Needle Aspiration and Subsequent Chemical Tinction, from the Lung Mass. Neoplastic Cells are Observed. Glandular Epithelial Cells were Seen Clustered, Pleomorphic, with Anisocytosis, Deep Blue Cytoplasm, Nuclear Molding and Anisokaryosis. There are Few Red Blood Cells, Lymphocytes and Macrophages on the Background.

B) The Samples were Obtained in the Necropsy. Lung Papillary Adenocarcinoma. Papillary Formations Lined by Cubic Cells in Mono and Bi-Layer. Macronuclei, with Some Obvious Nucleoli. Endoluminal Suppurative Inflammation. Low Mitotic Index (0-1). Haematoxylin and Eosin x 200.

C) The Samples were Obtained in the Necropsy. Chronic Splenitis. Hyperplasic Lymphoid Follicles with Adjacent Fibrosis in Red Pulp. Congestion and Hemorrhage. Haematoxylin and Eosin x 200.

D) The Samples were Obtained in the Necropsy. Segmentary Vacuolar Hepatosis and Cholestasis. Hepatocytes with Cytoplasmic Microvacuolated and Central Nucleous. Severe Cholestasis Center-Half and Lobular area. Slightly Dilated Central Veins. Sinusoids with Congestive Segmental Changes. Hepatosis Focus with Fat Vacuoles PeriLobulillar. Portal with a High Degree of Connective Tissue. Minimal Inflammatory Changes. Haematoxylin and Eosin x 200.

Despite having made only one cytoreductive surgery and after of drainage of the ascitic fluid accumulated in the abdominal cavity the animal improved in the first 48 hours after surgery. Within this 48 hours the animal was more active, had eaten some food and tolerated it and had minimal edema in the limbs. Postoperative chemotherapy with doxorubicin (Doxocris 50, LKM, Buenos Aires, Argentina), 30 $\mathrm{mg} / \mathrm{m}^{2} \mathrm{IV}$ was initiated immediately after surgery. The dog deteriorated 2 weeks after treatment and died. A complete necropsy (excluding the central nervous system) was performed. The right accessory lung lobe was replaced by a large tumor (11 x 6 × $4 \mathrm{~cm}$ ) previously diagnosed as a papillary pulmonary adenocarcinoma (Fig. 2 B). Due to the large size of the tumor, the CVC was almost completely compressed. The spleen had multiple redbrown nodules (Fig. 1 C) ranging from 0.5 to $1.3 \mathrm{~cm}$ in diameter; these splenic masses were histopathologically diagnosed as chronic splenitis (Fig. 2 C). The liver had vacuolar degenerative changes (Fig. 2 D). 


\section{Discussion}

BCLS is classified as primary, if the obstruction is the result of endoluminal venous lesion, such as thrombosis; or secondary, if the obstruction is caused by neighboring structures such as extrinsic compression or tumor invasion.

In this report we discuss a dog with secondary BCLS associated with a primary pulmonary adenocarcinoma. Secondary BCLS is rare in dogs, and has been reported due to liver lobe entrapment within the falciform ligament ${ }^{11}$, congenital hiatal hernia $^{12}$ and selected heart diseases ${ }^{3}$, 8 . In humans, other causes such as parasitic liver diseases or liver abscesses have also been reported in the literature. ${ }^{1}$

Obstruction of two or more major hepatic veins increases the sinusoidal pressure and reduces sinusoidal blood flow, producing clinical disease. The result of these hemodynamic changes is sinusoidal dilation and exudation of interstitial fluid, which diffuses through the liver capsule when the capacity of lymphatic drainage exceeds.

Ascites was classified as modified transudate, among other variables, due to the presence of red and inflammatory cells in the abdominal cavity. Regard of the cause, accumulation of fluid within the abdomen leads to additional fluid retention by the kidneys due to stimulatory effect on blood pressure hormones, firstly aldosterone. The sympathetic nervous system is also activated, and renin production is increased due to decreased perfusion of the kidney. Extreme disruption of the renal blood flow can lead to hepatorenal syndrome. Other complications of ascites include spontaneous bacterial peritonitis, due to decreased antibacterial factors in the ascitic fluid such as complement.

Thus, the ultrasonographic finding of liver congestion, pain in region of liver and ascites are the main signs of this disease presentation. Portal pressure increases and perfusion of the liver via portal vein is decreased. The combined effect of these changes in hepatic circulation on liver parenchyma is hypoxic damage of hepatocytes.

BCLS should be included in the list of differential diagnoses when animals present with: (1) Abrupt onset of ascites and painful hepatomegaly; (2) Massive ascites with relatively preserved liver functions; (3) Sinusoidal dilation in liver biopsy without heart disease; (4) Fulminant hepatic failure associated with hepatomegaly and ascites; (5) Unexplained chronic liver disease; (6) Hepatic thrombosis.

Liver biopsy exhibit marked hepatic congestion, liver cell waste and fibrosis predominantly located in the centrilobular area. ${ }^{1}$ The congestion can be seen in association with heart failure and/or restrictive pericardial disease. Liver biopsy is very important in differentiation of BCLS from veno-occlusive disease, which is characterized by nonthrombotic obstruction of hepatic venules by subendothelial swelling due to hurt of sinusoidal wall. Veno-occlusive disease could occurs also following administration of some toxic agents. ${ }^{13}$ Because obstruction of small hepatic veins without the involvement of large ones is included in the definition of BCLS, liver biopsy is fundamental for differentiation from venoocclusive disease. Bone marrow biopsy is helpful for diagnosis of primary myeloproliferative disorder. ${ }^{14,15}$

An odd feature of long-standing BCS is the development of multiple large regenerative nodules, some of them resembling chronic splenitis, as shown in Fig. 1 C. These nodules are a response to a focal loss of portal perfusion and hyperarterialization in areas with preserved hepatic or spleen vascularization. ${ }^{2}, 16$ This injury has been widely described in human patients with BCS, but has not yet been described in the veterinary literature. In our case, we found those lesions in the spleen (total of 17 nodules). 


\section{Conclusion}

Although no data are available on incidence of this disease in veterinary medicine, there is no doubt that this is a very serious illness despite its rare appearance. To our knowledge, this is one of a few cases of lung adenocarcinoma associated with BCLS, yet no other case has been highlighted in the literature review.

\section{Acknowledgements}

This study was funded by grants from the National Agency for Scientific and Technological Promotion (Argentina) and Quilmes National University. G.A.H., D.F.A., D.E.G. are members of CONICET (Argentina). We also thank the valuable collaboration of Dr. Sergio Ferraris in the sonographic diagnosis of this case.

\section{Abbreviations:}

BCS: Budd-Chiari syndrome

BCLS: Budd-Chiari like syndrome

CVC: caudal vena cava

\section{References}

1. Aydinli, M. \& Bayraktar, Y. (2007). "Budd-Chiari Syndrome: Etiology, Pathogenesis and Diagnosis," World Journal of Gastroenterology 2007; 13(19):2693-2696.

2. Valla, D. C. (2003). "The Diagnosis and Management of the Budd-Chiari Syndrome: Consensus and Controversies," Hepatology. 2003; 38:793-803.

3. van der Linde-Sipman, J. S. \& Stokhof, A. A. (1974). "Triple Atria in a Pup," Journal of The American Veterinary Medical Association. 1974; 165:539-541.

4. Stern, A., Fallon, R. K., Aronson, E. et al. (1986). "Cor Triatriatum Dexter in a Dog," The Compendium on continuing education for the practicing veterinarian. 1986;8: 401-414.
5. Miller, M. W., Bonagura, J. D., Dibartola, S. P. et al. (1989). 'Budd-Chiari Syndrome in Two Dogs,' Journal of the American Animal Hospital Association. 1989; 25:277-283.

6. Tobias, A. H., Thomas, W. P., Kittleson, M. et al. (1993). "Cor Triatriatum Dexter in Two Dogs," Journal of The American Veterinary Medical Association. 1993; 15:202(2):285-290.

7. Lombard, C. W. \& Goldschmidt, M. H. (1980). "Primary Fibroma in the Right Atrium of a Dog," Journal of Small Animal Practice. 1980; 21(8):439-448.

8. Atkins, C. E., badertscher, R. R., Greenlee, P. et al. (1982). 'Diagnosis of Intracardiac Fibrosarcoma Using Two Dimensional Echocardiography,' Journal of the American Animal Hospital Association. 1982; 20: 289-293.

9. Besso, J., Thollot, I. \& Bretonc, C. (1993). 'Un Casd'ascite Chez un Chien par Obstruction de la Venacave Caudale (Ascites in a Dog Due to Obstruction of the Caudal Vena Cava),' Le Point Veterinaire. 1993; 25:59-66.

10.Schoeman, J. P. \& Stidworthy, M. F. (2001). "Budd-Chiari-like Syndrome Associated with an Adrenal Phaeochromocytoma in a Dog," Journal of Small Animal Practice. 2001; 42(4):191-194.

11.Langs, L. L. (2009). "Budd-Chiari-Like Syndrome in a Dog Due to Liver Lobe Entrapment within the Falciform Ligament," Journal of the American Animal Hospital Association. 2009; 45(5):253-256.

12.Baig, M. A., Gemmill, T., Hammond, G. et al. (2006). "Budd-Chiari-Like Syndrome Caused by a Congenital Hiatal Hernia in a Shar-Pei Dog," Veterinary Record. 2006; 159(10):322-323. 
13.Gupta, S., Barter, S., Phillips, G. W. et al. (1987). "Comparison of Ultrasonography, Computed Tomography and 99mTc Liver Scan in Diagnosis of Budd-Chiari Syndrome," Gut. 1987;28(3):242-247.

14.Anger, B. R., Seifried, E., Scheppach, J. et al. (1989). "Budd-Chiari Syndrome and Thrombosis of Other Abdominal Vessels in the Chronic Myeloproliferative Diseases," Klinische Wochenschrift. 1989; 67:818-825.

15.De Stefano, V., Teofili, L., Leone, G. et al. (1997). "Spontaneous Erythroid Colony Formation as the Clue to an Underlying Myeloproliferative Disorder in Patients with Budd-Chiari Syndrome or Portal Vein Thrombosis," Semin Thromb Hemost. 1997; 23(5):411-418.

16. Renzulli, M., Lucidi, V., Mosconi, C. et al. (2011). "Large Regenerative Nodules in a Patient with Budd-Chiari Syndrome after TIPS Positioning while on the Liver Transplantation List Diagnosed by GdEOB-DTPA MRI," Hepatobiliary \& Pancreatic Diseases International. 2011; 10(4):439-442. 